\title{
ESTUDIO DEL PROPOLEOS DE SANTIAGO DEL ESTERO, ARGENTINA ${ }^{1}$
}

\author{
Lucrecia Lucía CHAILLOU ${ }^{2, *}$, Humberto Antonio HERRERA ${ }^{3}$, José Francisco MAIDANA ${ }^{4}$
}

\section{RESUMEN}

El objetivo principal de este trabajo fue determinar las características físico-químicas del propóleos de la provincia de Santiago del Estero. Las muestras se recolectaron de colmenas ubicadas en los departamentos Capital, Banda y Robles. Se determinaron: color, olor, sabor consistencia y aspecto. Las muestras por lo general, se presentaron en trozos irregulares con brillo, de estructura homogénea, consistencia dura. El color fue marrón oscuro, olor resinoso aromático y sabor picante. El porcentaje de impurezas mecánicas, cera y resinas se encuentran de valores normales. El índice de oxidación, las concentraciones de compuestos fenólicos y flavonoides y la actividad antibacteriana frente a Staphylococcus aureus permiten concluir que la calidad del propóleos de los departamentos estudiados es buena.

Palabras clave: fenoles; flavonoides; propóleos; resinas; extracto etanólico.

\section{RESUMO}

ESTUDO DE PRóPOLIS DE SANTIAGO DEL ESTERO, ARGENTINA. O objetivo principal deste trabalho foi a determinação das caracteristicas físico-químicas de própolis de Santiago del Estero. As amostras foram coletadas de departamentos Capital, Banda e Robles. A cor, o odor, o sabor, a consistência e a aparência externa foram analisados. As impurezas mecânicas, a cera, a resina, o índice da oxidação, os compostos fenólicos e os flavonóides foram testados. A atividade antimicrobiana foi determinada. As amostras foram apresentadas com estrutura homogênea, em parcelas irregulares brilhantes e a consistência era dura ou pouco macia na maioria dos casos. A cor era marrom-escura e o aroma resinoso aromático e o sabor picante. Porcentagem das impurezas, das ceras, da resina estavam entre valores normais. O indice de oxidação, as concentrações de compostos fenólicos e de flavonóides e a atividade antimicrobiana na Staphylococcus aureus permitam concluir que a qualidade dos própolis de departamentos Capital, Banda e Robles é boa.

Palavras-chave: compostos fenólicos; flavonóides; propolis; resinas; extrato etanólico.

\section{1 - INTRODUCCION}

El propóleos es un producto apícola de composición compleja [22]. Las abejas Apis mellifera L. lo obtienen por adición de cera y secreciones salivares al material resinoso, gomoso ó balsámico que recolectan de diversas plantas [5].

En la colmena, las abejas utilizan al propóleos con diversos fines, tales como: cerrar grietas, reducir al minimo las vías de acceso, recubrir y aislar restos de animales que se hayan introducido en la colmena, consolidar componentes estructurales, barnizar el interior de las celdillas con fines desinfectantes y evitar vibraciones [2, 6, 12].

Dentro de los productos apícolas, el propóleos se destaca por sus propiedades antibacterianas, fungicidas, antivirales, anestésicas, antiulcerosas, inmunoestimulantes, hipotensiva, citostática [2, 3, 6], antioxidantes [16, 20], fitoinhibidoras [2, 20] y anticariogénica [8].

${ }^{1}$ Recebido para publicação em 20/09/2001. Aceito para publicação em 30/10/2003 (000730).

2. Centro de Investigaciones Apícolas "Prof. Dr. Eduardo M. Bianchi". Facultad de Agronomía y Agroindustrias. Universidad Nacional de Sgo. del Estero. Avda. Belgrano 1912. Sgo. del Estero. C.P. 4200. Argentina. Tel: +54 3854509528 (int. 1703), Fax: +54 3854509585 E-mail: llchaillou@yahoo.com

3. Universidad Nacional de Sgo. del Estero. Avda. Belgrano 1912. Sgo. del Estero. C.P. 4200. Argentina. Tel: +54385 4509528, Fax: +54 3854222595 4. Centro de Investigaciones Apícolas "Prof. Dr. Eduardo M. Bianchi". Facultad de Agronomía y Agroindustrias. Universidad Nacional de Sgo. del Estero. Universidad Nacional de Sgo. del Estero. Avda. Belgrano 1912. Sgo. del Estero. C.P. 4200. Argentina.

*A quem a correspondência deve ser enviada.
En los últimos años se ha incrementado su utilización en medicina naturista y en veterinaria $[7,15]$. Es por lo tanto una materia prima valiosa para la industria farmacéutica, de cosméticos y de alimentos [2].

De acuerdo con numerosos estudios, se han determinado que los constituyentes principales son las ceras, resinas, bálsamos, aceites esenciales, polen, además de impurezas mecánicas $[2,4,19]$. La proporción de los mismos es variable y depende de la época de recolección, así como también del origen vegetal de la resina y de la raza de las abejas [11].

El objetivo principal de este trabajo fue determinar en el propóleos, de los departamentos que concentran la mayor cantidad de colmenas: Capital, Banda y Robles de la provincia de Santiago del Estero, República Argentina, aspectos de su calidad que involucran los caracteres organolépticos, es decir: aspecto, estructura, consistencia, color, olor, sabor; y la composición del mismo, para ello se determinó el contenido de impurezas mecánicas, cera, resina, compuestos fenólicos y flavonoides. También se midieron el punto de fusión y el índice de oxidación, y se determinó la actividad antibacteriana contra cepas de Staphylococcus aureus, coagulasa positivo.

\section{2 - MATERIALES Y METODOS}

\section{1 - Material}

\subsection{1 - Propóleos}

Se recolectaron, durante los meses de Septiembre y Octubre del año 2000, por raspado, muestras de pro- 
póleos, de aproximadamente 100g, de colmenas ubicadas en localidades de los departamentos Capital, Banda y Robles de la provincia de Santiago del Estero, República Argentina. Las muestras se almacenaron en el laboratorio refrigeradas y al abrigo de la luz. Previamente al análisis fueron trituradas, hasta reducir considerablemente su granulometría y homogeneizadas. Se analizaron por triplicado.

\subsection{2 - Extracto etanólico de propóleos al 1\%p/v}

Se prepara con una muestra de propóleos de $1 \mathrm{~g}$, triturada y homogeneizada. La misma se extrajo con $100 \mathrm{~mL}$ de etanol de $96^{\circ}$, en porciones de $10 \mathrm{~mL}$ a $50^{\circ} \mathrm{C}$, se filtró para retener las impurezas. El extracto se conserva refrigerado, en frascos de color ámbar.

\section{2 - Métodos}

\subsection{1 - Caracteres organolépticos}

El aspecto, estructura, consistencia, color, olor y sabor se determinaron mediante pruebas sensoriales descriptivas, realizadas por un grupo de 10 personas semientrenadas que calificaron los atributos, en sesiones de 5 muestras cada una, utilizando escalas de intervalo [1]. Las escalas fueron de 2 puntos (homogénea y heterogénea) para la estructura; de 5 puntos para el aspecto (polvo, granulado, trozos irregulares opacos, trozos irregulares con poco brillo, trozos irregulares con brillo); de 3 puntos para la consistencia (blanda, poco blanda y dura), para el olor (resinoso suave, resinoso, resinoso aromático) y para el sabor (insípido, dulce, picante) y de 15 puntos para el color (distintas tonalidades de marrón) [9]. Para el análisis se colocaron las muestras, codificadas, en frascos de vidrio con tapa a rosca y se conservaron a $25^{\circ} \mathrm{C}$ durante 3 días, al cabo de los cuales se evaluaron, primeramente, el olor y el sabor y luego el resto de los caracteres comparando cada muestra con controles adecuados. El aspecto y la estructura se determinaron por observación visual, la consistencia por opresión de la muestra con los dedos, el color mediante la comparación con una escala de colores.

\subsection{2 - Punto de fusión}

Se determinó utilizando un tubo capilar, en el cual se coloca la muestra, adosado a un termómetro, ambos se calentaron en un baño de glicerina. Se registró la temperatura de comienzo y finalización de la fusión, para el análisis estadístico se tomó el promedio de ambas temperaturas.

\subsection{3 - Indice de oxidación}

Este índice se define como el tiempo de decoloración, medido en segundos, de una solución de permanganato de potasio $0.1 \mathrm{~N}$ por acción de los compuestos oxidantes del propóleos. Para ello, se trituró la muestra con alcohol de $96^{\circ}$, luego se llevó a $100 \mathrm{~mL}$ con agua destilada y se filtró. Se tomó una alícuota del filtrado, se acidificó con ácido sulfúrico al $20 \%$ y se añadió una gota de permanganato de potasio
0.1N. Se midió con cronómetro el tiempo de decoloración [18].

\subsection{4 - Impurezas mecánicas}

La muestra se trató sucesivamente con: n-heptano, a $50^{\circ} \mathrm{C}$; cloroformo-acetona $(2: 1)$ y alcohol etílico absoluto. Luego del agregado de cada solvente se filtró la mezcla a través de un papel de filtro de filtración rápida, previamente desecado (a $100-105^{\circ} \mathrm{C}$ ) y pesado. El residuo que quedó retenido en el papel de filtro, se llevó a estufa, a $100-105^{\circ} \mathrm{C}$, hasta peso constante. Por diferencia de peso con el papel sin residuo se determinó el contenido de impurezas, que se expresa en porcentaje ( $g / g)$ [13].

\subsection{5 - Cera}

El contenido de cera se determinó por extracción con éter de petróleo (intervalo de destilación $60-80^{\circ} \mathrm{C}$ ) en un extractor Soxhlet [14]. Se expresa en porcentaje (g/g).

\subsection{6 - Resina}

Se extrajeron las resinas, de muestras de $10 \mathrm{~g}$, colocadas en frascos con tapa de color ámbar, con $100 \mathrm{~mL}$ de etanol de $96^{\circ}$, a $40^{\circ} \mathrm{C}$, con agitación diaria de 30 minutos, durante 72 horas. Luego el frasco se llevó a $0^{\circ} \mathrm{C}$ durante 4 horas, se filtró para retener las ceras e impurezas mecánicas. Se eliminó el solvente del filtrado por evaporación suave y se determinó el contenido de resinas por gravimetría, que se expresa en porcentaje (g/g), en base a la técnica descripta por PECHEANSKI [17].

\subsection{7 - Compuestos fenólicos}

Los compuestos fenólicos se determinaron mediante la técnica que utiliza el reactivo de Folin - Ciocalteau. Para ello se trató el extracto etanólico de propóleos $(0.1 \% \mathrm{v} / \mathrm{v})$ con el reactivo de Folin - Ciocalteau, se agregó carbonato de sodio al 15\% y se llevó a volumen $(20 \mathrm{~mL})$ con agua destilada. Se colocó luego en baño de agua a $50^{\circ} \mathrm{C}$ durante 5 minutos, se enfrió y se determinó la absorbancia a $765 \mathrm{~nm}$, mediante un espectrofotómetro. La concentración de fenoles totales se obtuvo a partir de la curva de calibración construida con concentraciones conocidas de soluciones patrón de ácido gálico [13]. Se expresa en porcentaje (mg/g).

\subsection{8 - Flavonoides}

A una alícuota de extracto etanólico de propóleos $0.1 \%(\mathrm{v} / \mathrm{v})$, se le agregó $0.5 \mathrm{~mL}$ de la solución de cloruro de aluminio al $5 \%(\mathrm{p} / \mathrm{v})$ y se llevó a volumen $(25 \mathrm{~mL})$ con metanol. Se leyó la absorbancia a $425 \mathrm{~nm}$ mediante un espectrofotómetro utilizándose metanol como blanco [18]. La concentración de flavonoides se obtuvo mediante la curva de calibrado que se construyó utilizando soluciones de concentración conocida de quercetina. Se expresa en porcentaje (mg/g).

\subsection{9 - Actividad antibacteriana}

Se determinó la actividad antibacteriana de extractos alcohólicos de propóleos al 10\% (p/v), por el méto- 
do de difusión en doble capa de medio agar nutritivo frente a una cepa de Staphylococcus aureus, coagulasa positivo. Las placas se incubaron a $37^{\circ} \mathrm{C}$ durante 24 horas. Se midió con calibre el diámetro del halo de inhibición [2].

\section{3 - RESULTADOS Y DISCUSIÓN}

\section{1 - Caracteres organolépticos}

El $100 \%$ de las muestras presentaron estructura homogénea, el $45 \%$ presentó un aspecto de trozos irregulares con brillo, el 30\% uno de trozos irregulares con poco brillo y el resto como trozos irregulares opacos.

Los ensayos de consistencia mostraron que el $45 \%$ de las muestras eran poco blandas, el $40 \%$ duras y solamente el $15 \%$ blandas.

Con respecto al color, el 65\% de las muestras presentaron color marrón oscuro, un 20\% marrón claro con tintes amarillos, un 10\% marrón con tintes castaños y un 5\% marrón claro con tintes castaños el olor del $75 \%$ de las mismas fue resinoso y el sabor de la totalidad de los propóleos analizados fue picante.

\section{2 - Punto de fusión}

Los resultados se muestran en la Tabla 1, que incluye el valor medio de temperaturas de fusión.

TABLA 1. Temperatura media de Fusión

\begin{tabular}{c|l|c}
\hline \multirow{2}{*}{ Departamento } & \multicolumn{1}{|c}{ Localidad } & $\begin{array}{c}\text { Temperatura media de fusión } \\
\left({ }^{\circ} \mathrm{C}\right)\end{array}$ \\
& Maco & 69.5 \\
& San Benito & 74.5 \\
CAPITAL & San Pedro & 64.0 \\
& Sgo. del Estero & 64.0 \\
& Vuelta de la Barranca & 66.0 \\
& Yanda & 68.0 \\
\hline \multirow{5}{*}{ BANDA } & Antajé & 74.5 \\
& Clodomira & 66.0 \\
& Colonia Gamara & 73.0 \\
& El Simbolar & 69.5 \\
& Guaycurú & 69.5 \\
& Jumi Pozo & 70.0 \\
& La Banda & 68.5 \\
& San Isidro & 80.5 \\
& Tramo 16 & 72.5 \\
\hline \multirow{6}{*}{ ROBLES } & Beltrán & 73.0 \\
& Colonia & 75.5 \\
& El Simbolar & 72.0 \\
& Forres & 72.0 \\
& El Barrial & 70.0 \\
\hline
\end{tabular}

El valor medio de la temperatura de fusión obtenido para el departamento Robles fué de $72.5^{\circ} \mathrm{C}$, para Banda $70.6^{\circ} \mathrm{C}$ y $67.7^{\circ} \mathrm{C}$ para Capital. Las temperaturas de fusión se encuentran dentro de $\operatorname{los} 60$ a $100^{\circ} \mathrm{C}$, establecidos como el rango más frecuente en la bibliografía [11].

\section{3 - Impurezas mecánicas, cera y resinas}

Los contenidos de impurezas mecánicas, cera y resinas, expresados en porcentaje (g/g), se muestran en la Tabla 2.
TABLA 2. Contenidos de impurezas mecánicas, cera y resinas

\begin{tabular}{|c|c|c|c|c|}
\hline Departamento & Localidad & $\begin{array}{c}\text { Cera } \\
(\%)\end{array}$ & $\begin{array}{c}\text { Impurezas } \\
\text { mecánicas (\%) }\end{array}$ & $\begin{array}{c}\text { Resinas } \\
(\%)\end{array}$ \\
\hline \multirow{6}{*}{ CAPITAL } & Maco & 37.480 & 17.165 & 45.530 \\
\hline & San Benito & 23.077 & 38.963 & 36.932 \\
\hline & San Pedro & 18.819 & 37.139 & 44.030 \\
\hline & Sgo. del Estero & 36.170 & 15.561 & 48.271 \\
\hline & Vuelta de la Barranca & 33.683 & 16.529 & 41.659 \\
\hline & Yanda & 38.807 & 21.362 & 39.831 \\
\hline \multirow{9}{*}{ BANDA } & Antajé & 20.031 & 26.179 & 53.410 \\
\hline & Clodomira & 33.801 & 23.412 & 42.779 \\
\hline & Colonia Gamara & 34.299 & 22.757 & 42.930 \\
\hline & El Simbolar & 17.042 & 30.001 & 41.046 \\
\hline & Guaycurú & 20.975 & 20.975 & 56.719 \\
\hline & Jumi Pozo & 36.675 & 14.933 & 48.062 \\
\hline & La Banda & 38.420 & 26.536 & 35.050 \\
\hline & San Isidro & 19.800 & 37.305 & 41.354 \\
\hline & Tramo 16 & 36.349 & 12.499 & 54.144 \\
\hline \multirow{5}{*}{ ROBLES } & Beltrán & 30.560 & 20.188 & 49.251 \\
\hline & $\begin{array}{l}\text { Colonia } \\
\text { El Simbolar }\end{array}$ & 49.083 & 9.979 & 40.605 \\
\hline & Forres & 27.492 & 27.675 & 44.820 \\
\hline & El Barrial & 19.345 & 31.421 & 48.871 \\
\hline & Los Romanos & 29.045 & 30.673 & 40.272 \\
\hline
\end{tabular}

El valor medio del contenido de impurezas mecánicas fue $24.063 \%$. Este valor es menor que el admitido por el reglamento brasileño (40\%) [18]. La media aritmética del contenido de impurezas mecánicas de $24.063 \%$ está por debajo del máximo de 30\% propuesto por MAIDANA [9] en un estudio por regiones fitogeográficas, pero por encima del promedio $15.15 \%$, de la región Noroeste de Argentina [10]. Si se analiza el contenido de impurezas, para cada departamento, se tiene: $24.453 \%$ para Capital y $23.822 \%$ y $23.987 \%$ para Banda y Robles respectivamente.

En cuanto al contenido de cera, los departamentos Capital, Banda y Robles, presentaron: 31.339\%, $28.599 \%$ y $31.050 \%$, respectivamente. El valor medio fué de $30.048 \%$, que es ligeramente superior al máximo del 30\% y al promedio de $23.83 \%$ para el Parque Chaqueño, establecidos por MAIDANA [9]. Sin embargo, este valor es ligeramente inferior al 36.19\%, encontrado por MALDONADO [10] para la región Noroeste. Estos valores superan el máximo de 25\% admitido en las especificaciones brasileñas [18].

El porcentaje relativamente bajo de resinas de $44.770 \%$, se debe probablemente, al alto contenido de ceras y a que las especies vegetales circundantes al colmenar no son muy ricas en resinas. Es menor que el $52.86 \%$ determinado en el Parque Chaqueño por MAIDANA [9], y ligeramente inferior al $44.14 \%$ determinado por MALDONADO [10] para la región Noroeste. Este valor relativamente bajo, comparado con el Parque Chaqueño, se debe al elevado contenido de ceras de los propóleos santiagueños, como puede observarse en la Tabla 2 y probablemente a que la vegetación circundante al colmenar no produce resinas en abundancia. El mayor contenido de resinas se obtuvo en el departamento Banda seguido por Robles y Capital.

\section{4 - Indice de oxidación, compuestos fenólicos y flavonoides}

En la Tabla 3 se muestran los índices de oxidación que se obtuvieron para los tres departamentos 
estudiados. El rango de valores del índice de oxidación osciló entre los 4 y 18 segundos, el promedio fue de 9.8 segundos, estos valores son inferiores al valor máximo de 22 segundos admitidos en los reglamentos internacionales [2], pero superiores a los 4.9 segundos determinados para la Región Noroeste por MALDONADO [10], esto se debe posiblemente al que el contenido de ceras e impurezas son mayores que el que presentan los propóleos de la región Noroeste, así como también a un menor contenido de compuestos fenólicos.

TABLA 3. Indice de oxidación, compuestos fenólicos y flavonoides

\begin{tabular}{|c|c|c|c|c|}
\hline Departamento & Localidad & $\begin{array}{c}\text { Indice de } \\
\text { oxidación (s) }\end{array}$ & \begin{tabular}{|c|}
$\begin{array}{c}\text { Compuestos } \\
\text { fenólicos } \\
(\%, \mathrm{mg} / \mathrm{g})\end{array}$ \\
\end{tabular} & $\begin{array}{c}\text { Flavonoides } \\
(\%, \mathrm{mg} / \mathrm{g})\end{array}$ \\
\hline \multirow{6}{*}{ CAPITAL } & Maco & 12 & 13.260 & 2.024 \\
\hline & San Benito & 4 & 20.993 & 2.179 \\
\hline & San Pedro & 11 & 10.803 & 0.633 \\
\hline & Sgo. del Estero & 14 & 8.615 & 1.103 \\
\hline & Vuelta de la Barranca & 18 & 15.863 & 1.405 \\
\hline & Yanda & 15 & 10.681 & 0.943 \\
\hline \multirow{9}{*}{ BANDA } & Antajé & 4 & 22.102 & 1.464 \\
\hline & Clodomira & 9 & 16.531 & 1.896 \\
\hline & Colonia Gamara & 15 & 11.483 & 0.915 \\
\hline & El Simbolar & 10 & 15.054 & 1.053 \\
\hline & Guaycurú & 3 & 21.464 & 1.899 \\
\hline & Jumi Pozo & 5 & 25.267 & 1.065 \\
\hline & La Banda & 9 & 17.855 & 1.528 \\
\hline & San Isidro & 8 & 16.567 & 1.794 \\
\hline & Tramo 16 & 4 & 21.538 & 0.833 \\
\hline \multirow{5}{*}{ ROBLES } & Beltrán & 15 & 16.246 & 1.214 \\
\hline & $\begin{array}{l}\text { Colonia } \\
\text { El Simbolar }\end{array}$ & 18 & 4.251 & 0.230 \\
\hline & Forres & 5 & 17.929 & 1.005 \\
\hline & El Barrial & 5 & 19.137 & 1.332 \\
\hline & Los Romanos & 12 & 12.718 & 1.479 \\
\hline
\end{tabular}

Las muestras analizadas presentaron como contenidos medios de compuestos fenólicos y flavonoides $15.918 \%$ y $1.300 \%$, respectivamente. El contenido de estos compuestos fenólicos supera el valor mínimo de $5 \%$, establecido en el reglamento brasileño de calidad de propóleos [18], y el porcentaje de flavonoides permite clasificar a los propóleos ensayados como de tenor medio de flavonoides de acuerdo con éste reglamento [18]. El contenido promedio de compuestos fenólicos totales que se encontró en los propóleos analizados fue de 15.918\%, y es superior al $12.33 \%$ del Parque Chaqueño, establecido por MAIDANA [9], pero inferior al $16.07 \%$ de la región Noroeste, obtenido por MALDONADO [10], esta diferencia se debe probablemente a las diferentes especies botánicas que las abejas utilizan como fuente de resinas.

Los propóleos del departamento Banda presentan el mayor contenido promedio de fenoles totales (18.651\%), seguido por Robles y Capital con 14.056 y $13.369 \%$, respectivamente.

En cuanto a los flavonoides, los de la región Noroeste, con un contenido de $7.31 \%$ informado por MALDONADO [10], superan considerablemente al valor promedio de $1.300 \%$ de los propóleos analizados. Esta diferencia probablemente sea también una consecuencia de las diferentes especies botánicas que las abejas de una y otra región utilizan como fuente de resinas. Capi- tal y Banda presentan propóleos con los mayores contenidos de flavonoides, 1.383 y $1.381 \%$, respectivamente.

\subsection{Actividad antibacteriana}

Los diámetros de los halos de inhibición se muestran en la Tabla 4.

TABLA 4. Diámetro de los halos de inhibición

\begin{tabular}{c|l|c}
\hline Departamento & \multicolumn{1}{|c}{ Localidad } & $\begin{array}{c}\text { Diámetro del halo de } \\
\text { inhibición }(\mathrm{mm})\end{array}$ \\
\hline \multirow{5}{*}{ CAPITAL } & Maco & 12.067 \\
& San Benito & 13.267 \\
& San Pedro & 10.050 \\
& Sgo. del Estero & 7.000 \\
& Vuelta de la Barranca & 12.076 \\
& Yanda & 14.033 \\
\hline \multirow{5}{*}{ BANDA } & Antajé & 12.367 \\
& Clodomira & 14.100 \\
& Colonia Gamara & 9.567 \\
& El Simbolar & 0.000 \\
& Guaycurú & 12.000 \\
& Jumi Pozo & 17.067 \\
& La Banda & 9.400 \\
& San Isidro & 16.100 \\
& Tramo 16 & 12.000 \\
\hline \multirow{5}{*}{ ROBLES } & Beltrán & 9.867 \\
& Colonia El Simbolar & 0.000 \\
& Forres & 10.633 \\
& El Barrial & 11.033 \\
& Los Romanos & 10.967 \\
\hline
\end{tabular}

El diámetro promedio de halo de inhibición fue $10.680 \mathrm{~mm}$, esto indica la existencia de actividad antibacteriana de los extractos etanólicos al 10\% (p/v) de concentración. El diámetro del halo de inhibición promedio que se determinó fue de $10.680 \mathrm{~mm}$. Este es ligeramente inferior al detectado en Santiago del Estero, dentro de la Región Noroeste, en un estudio de actividad antibacteriana de propóleos realizado por TABERA et al. [21] y a lo expresado por la técnica cubana de actividad antimicrobiana del propóleos, que indica un diámetro de halo de inhibición de $12 \mathrm{~mm}$, de acuerdo con ASÍS [2]. Sin embargo si se analizan las muestras individualmente, un $50 \%$ de las mismas cumplen con ésta medida de $12 \mathrm{~mm}$ de diámetro. El diámetro del halo de inhibición depende del contenido de flavonoides de los extractos ensayados y como el mismo es inferior al de la región Noroeste, el diámetro promedio de los departamentos estudiados es mas bajo.

En la Tabla 4 se puede observar que los propóleos de los departamentos Capital y Banda presentan valores promedio de halo de inhibición muy próximos, siendo los menos variables las muestras procedentes del primer departamento mencionado.

\section{4 - CONCLUSIONES}

- Por lo general, los propóleos de los departamentos Capital, Banda y Robles, de la provincia de Santiago del Estero, presentan estructura homogénea, aspecto de trozos irregulares con brillo, de consistencia dura a poco blanda. El color es marrón oscuro, olor resinoso y sabor picante. 
- $\quad \mathrm{El}$ punto de fusión oscila entre 64 y $80.5^{\circ} \mathrm{C}$.

- El contenido de impurezas mecánicas y resinas se encuentra dentro de los límites admitidos por la legislación brasileña, sin embargo el contenido de ceras es ligeramente superior al valor admitido.

- $\quad$ El índice de oxidación, el contenido de fenoles totales y flavonoides indican que los propóleos son de buena calidad, además los mismos presentan actividad antibacteriana sobre cepas de Staphylococcus aureus, coagulasa positivo.

- El departamento Banda se destaca por el contenido elevado de fenoles totales y flavonoides, que son los principios biológicamente activos. Asimismo, la calidad de los propóleos de los departamentos Capital y Robles es buena.

\section{5 - REFERENCIAS BIBLIOGRAFICAS}

[1] ANZALDUA-MORALES, A. 1994. La evaluación sensorial de los alimentos en la teoría y la práctica. Editorial ACRIBIA S. A. Zaragoza. España.

[2] ASÍS, M.1989. Propóleos: el oro púrpura de las abejas. Centro de Información y Documentación Agropecuaria. Editorial CIDA. La Habana. Cuba.

[3] BANKOVA,V.S.; POPOV,S.S.; MARENKOV,W.L. High perfomance liquid chromatographic analysis of flavonoids from propolis. Journal of Chromatography, n. 242, p.135-143, 1982.

[4] BURDOCK, G.A.. Review of te biological properties and toxicity of bee propolis. Food and Chemical Toxicology n. 36, p. 347-363, 1998.

[5] FRANCO,T.T.;KUREBAYASHI, A.K. Isolamento de principios ativos da própolis por cromatografía em papel bidimensional e dosamento espectrofotometrico. Revista del Instituto Adolfo Lutz. n. 46 (1/2), p.81-86, 1986.

[6] GONZÁleZ Guerra, A.; Bernal MÉndeZ, R. Propóleos: un camino hacia la salud. Editorial Pablo de la Torriente. La Habana. Cuba, 1997. Cap.1, p. 8-28.

[7] IORISH, N. Propóleos. Investigaciones científicas y opiniones a cerca de su composición, caracteristicas y utilización con fines terapéuticos. Comisión Permanente de Tecnología y Utillaje apícolas. Editorial Apimondia. Bucarest. Rumania, 1975. Capítulo IV: Empleo del Propóleos en medicina, p. 89-90.

[8] KOO, H.; ROSALEN, P. L.; CURY, J. A.; PARK, Y. K.; IKEGAKI, M.; SATTLER, A. Effect of Apis mellifera L. Propolis from Two Brasilian Regions on Caries Development in Desalivated Rats. Caries Research. n. 33, p. $393-400,1999$

[9] MAIDANA, J. F. Características Físico-químicas del Propóleos de la República Argentina. Tucumán, 1997. 250 p. Tesis Doctoral, para obtener el título de Doctor en Biología. Facultad de Agronomía y Zootecnia. Universidad Nacional de Tucumán (UNT). Tucumán. Argentina.

[10] MALdonado, L.. Perfil de los Propóleos argentinos. Actas del Congreso Internacional de Propóleos. Buenos Aires. Argentina. p. 11-12, 2000.

[11] MEDA,A.C.;MATTOS MEDA, A.P.. Própolis um bem da humanidade- Produçao e controle. Anales del $X$ Congreso Brasilero de Apicultura. Pousada do Rio Quente Do. Brasil. p. 46-50, 1994.
[12] MORSE, G. D. A cerca del Propóleos. Su utilización en la colmena. Propóleos. Investigaciones científicas y opiniones a cerca de su composición, características y utilización con fines terapéuticos. Comisión Permanente de Tecnología y Utillaje apícolas. Editorial Apimondia. Bucarest. Rumania. Capítulo I: Generalidades, p. 59-63, 1975.

[13] NORMA RAMAL CUBANA SOBRE ESPECIFICACIONES DE CALIDAD DE PROPÓlEOS. NRAG 870-88, Dirección de Normalización, Metrología y Control de Calidad del Ministerio de Agricultura de Cuba. Citada en Propóleos: el oro púrpura de las abejas. ASíS M. Centro de Información y Documentación Agropecuaria. Editorial CIDA. La Habana. Cuba, p. 127-148, 1989.

[14] NORMA RAMAL DE HUNGRÍA MSZ080184-79 del Ministerio de Agricultura y Alimentos de la Rep. Popular de Hungría. Citada en Propóleos: el oro púrpura de las abejas. ASíS $M$. Centro de Información y Documentación Agropecuaria. Editorial CIDA. La Habana. Cuba, p. 116-117

[15] PARK, Y. K.; KOO, M. H.; SATO, H. H.; CONTADO, J. L.. Estudo de alguns componentes da própolis coletada por Apis mellifera L. no Brasil. Arq. Biol. Tecnol., v. 38 n. 4, p. 1253-1259, 1995.

[16] PARK, Y. K.; IKEGAKI, M.;DA SILVA, J. A.; FREIRE, N. M.;. Estudo da preparaçao dos extratos de própolis e suas aplicaçoes. Cienc. Tecnol. Aliment., v. 18, n. 3, p. 257-362, 1998.

[17] PECHEANSKI, A. N. Tratamientos de algunas enfermedades con soluciones de propóleos. Propóleos. Investigaciones científicas y opiniones a cerca de su composición, características y utilización con fines terapéuticos. Comisión Permanente de Tecnología y Utillaje apícolas. Editorial Apimondia. Bucarest. Rumania. Capítulo IV: Empleo de propóleos en medicina, p. 100-101, 1975.

[18] REGULAMENTOS TÉCNICOS PARA FIXAÇAO DE IDENTIDADES E QUALIDADE DE PRÓPOLIS. Mensagem Doce. Apacame. n. 52. 1999.

[19] RIBEIRO CAMPOS, M. G.;. Contribuçao para o Estudo do Mel, Polen, Geleia Real e Própolis. Boletim da faculdade de Farmacia de Coimbra. Portugal. v. 11, n. 2,. p. 17-47, 1987.

[20] STANGACIU, S. Composición y Propiedades del Propóleos. Apimondia. Apiacta n. 33, p. 71-77, 1998.

[21] TABERA, A; BEDASCARRASBURE, E.; MALDONADO, L.; ALVAREZ, A.; VAN DER HORST, A.. Actividad Antibacteriana de propóleos argentinos enfrentados a Staphylococcus aureus. Actas del Congreso Internacional de Propóleos. Buenos Aires. Argentina. p. 97, 2000.

[22] WOISKY, R. G.; GIESBRECHT, A.; SAlatino, A. Actividade Antibacteriana de uma Formulaçao preparada a partir de Própolis de Apis mellífera L. Actas del IV Congreso Iberoamericano de Apicultura. I Foro Expo-Comercial Internacional de Apicultura. Rio Cuarto. Córdoba. Argentina. p. 213-216, 1994.

\section{6 - AGRADECIMIENTOS}

A los Señores Técnicos Apicultores: Ana Chazarreta, Fernando Brandán, Santiago E. Traine; a la Sra. Directora de la Escuela de Apicultura Fidela S. de Smith: Dora Leiva de Herrera. 